\title{
REVIEW
}

\section{Sprouty proteins: modified modulators, matchmakers or missing links?}

\author{
G R Guy, R A Jackson, P Yusoff and S Y Chow \\ Signal Transduction Laboratory, Institute of Molecular and Cell Biology, 61 Biopolis Drive, Proteos, Room 3-14B, Singapore, Republic of Singapore 138673 \\ (Correspondence should be addressed to G R Guy; Email: mcbgg@imcb.a-star.edu.sg)
}

\begin{abstract}
Sprouty proteins are involved in organogenesis, particularly during the branching of endothelial tubes, and existing evidence suggests that Sprouty's point of action lies downstream of receptor signaling to inhibit the activation of the central Ras/Erk pathway. How Sprouty proteins accomplish their inhibitory action and whether they interact with other signaling pathways are significant questions. Sprouty proteins are devoid of any recognizable protein interaction domain, and clues as to how they function have been mainly derived from screening for interacting partners. Conserved across all the Sprouty proteins are three sequences: a Cbl-tyrosine kinase-binding (TKB) binding motif centered on an obligatorily phosphorylated tyrosine (Y55 in Sprouty2), a serine-rich motif (SRM) and a cysteine-rich domain (CRD). With the exception of a handful of proteins that bind to the N-terminus, most of the binding to Sprouty occurs via the
\end{abstract}

CRD, predominantly by serine/threonine kinases that target sites within the SRM on Sprouty. Some of the resultant increase in phosphorylation is opposed by activated protein phosphatase 2A that binds to the N-terminal Cbl-TKB binding motif. Significantly, two ubiquitin E3 ligases also bind to the N-terminus of Sprouty: $\mathrm{c}-\mathrm{Cbl}$ binds with high affinity to the TKB binding motif and SIAH2 binds constitutively to a different site; both proteins are able to direct the ubiquitination of Sprouty proteins and its destruction. The collective evidence points to Sprouty proteins as being substantially covalently-modified to control its location, stability, association, and destruction. With such stringent control of the Sproutys, the main question is what key proteins does this facilitator bring together?

Journal of Endocrinology (2009) 203, 191-202

\section{Introduction}

The nature and number of intracellular pathways responsible for transmitting cues from the external environment into a physiological response was literally a 'black box' of knowledge deficiency, 20 years ago. Since then, the field of cell signaling has occupied $30-50 \%$ of the cell biology research literature, and is gaining an ever-expanding insight into the complexity of transduction pathways employed by the cell. Marrying information garnered from Caenorhabditis elegans and Drosophila, the first signaling pathway - where a rational and continuous connection was made between growth factor/ receptor engagements to the transcription of a subset of genes downstream - was the receptor tyrosine kinase (RTK)/ Ras/ERK pathway. Although this pathway achieved fame as the first to be delineated, it still maintains a high profile in signaling (Pearson et al. 2001, Roux \& Blenis 2004, Murphy \& Blenis 2006), and ensuing research has demonstrated that each of the core components in the pathway is subjected to multiple positive and negative signals and can be clustered together by a range of docker and scaffold proteins in a temporal or spatial manner within the cell (Brown \& Sacks 2009). Moreover, many aberrations in this pathway are most epitomized by cancer, particularly in three of the central players, RTKs (e.g. epidermal growth factor receptor, EGFR), Ras and Raf. Each of these proteins has a high gain-of-function mutational incidence (Dhillon et al. 2007) and therefore, inhibitors of the RTK/Ras/ERK pathway are strategically placed to control this indisputably key pathway in cellular function. Two families of inhibitors of the pathway have emerged in the last decade, termed Sprouty and Sprouty-related Ena/VASP homology 1 (EVH1) domain containing protein (SPRED), and are the focus of research for a number of groups.

\section{Sprouty and Spred}

The founding member of the Sprouty (Spry) family was discovered in a screen for genes in Drosophila that were responsible for shaping the developing trachea (Hacohen et al. 1998). The sole Drosophila Spry protein was demonstrated to inhibit the RTK/Ras/ERK pathway downstream of several 
different growth factors (Casci et al. 1999). Subsequent studies revealed four mammalian SPRY proteins with one of them, SPRY2, playing a similar role to dSpry in modeling the branching of the mammalian lung (de Maximy et al. 1999, Tefft et al. 1999). There were few clues from the sequences of the SPRY proteins as to their likely mode of action, with initial analyses indicating no likely enzyme motifs and no conserved protein-protein interaction domains. The family members contained a conserved but novel cysteine-rich domain (CRD) that was later found reiterated in the SPRED family of proteins (Wakioka et al. 2001).

Sprouty proteins are ubiquitously expressed in the developing embryo as well as in adult tissues, with the exception of SPRY3, which has a more restricted distribution in the brain and the testis (Minowada et al. 1999, Leeksma et al. 2002). Evidence from gene knock-out studies demonstrate SPRY's role in the formation of a number of tissues and organs during mammalian development (summarized in Supplementary Table 1, available in the online version of the Journal of Endocrinology at http://joe.endocrinology-journals.org/cgi/content/full/ JOE-09-0110/DC1). Presently, the major role assigned to SPRY is to inhibit Ras/Raf/ERK signaling and SPRY proteins have been shown to do this downstream of a wide range of growth factor stimuli, including fibroblast growth factor (FGF), vascular-endothelial growth factor, plateletderived growth factor (PDGF), hepatocyte growth factor, glial-derived growth factor, and nerve growth factor (NGF; reviewed in Guy et al. 2003, Li et al. 2003, Kim \& Bar-Sagi 2004, Mason et al. 2006, Cabrita \& Christofori 2008). Interestingly, the inhibitory function of SPRY is both cell and ligand specific, as SPRY does not inhibit MAPK activation in response to EGF signaling (Sasaki et al. 2001). Despite a mound of literature dedicated to SPRY's activity, a universal mechanism as to how SPRY accomplishes its action is yet to be confirmed, and most of the evidence comes from cell culture and over-expression-derived data. While SPRY proteins exercise their effects by directly interacting with other proteins, their own levels can also be controlled at various points of transcription and translation. Indeed, SPRY1 levels in myocardial fibroblasts can be controlled at the level of translation by microRNA-21 leading to increased MAP kinase levels concomitant with the onset of cardiovascular disease (Thum et al. 2008).

Owing to its shared interaction domain with Sprouty proteins, a brief introduction of the SPRED protein family is necessary (a more comprehensive review can be found elsewhere (Bundschu et al. 2007)). Prior to the discovery of dSpry, a gene called ae33 was discovered in a screen for Drosophila eye development (DeMille et al. 1996). The predicted protein from this transcript showed strong homology to enabled (Ena) and vasodilator-stimulated phosphoprotein (VASP) in its N-terminus, and a novel cysteine-rich (cys-rich) region in its C-terminus. Some years later, in a yeast two-hybrid screen for interacting partners to the active kinase domain of $\mathrm{c}-\mathrm{Kit}$ and $\mathrm{c}-\mathrm{Fms}$ receptors,
Wakioka et al. (2001) isolated a novel protein from a mouse osteoclast cDNA library that they designated SPRED1 in reference to its $\mathrm{N}$-terminal EVH1 domain and its Cys-rich C-terminus reminiscent of the CRD that was at the time unique to Sprouty.

There are four mammalian SPRED proteins: SPRED1-3 and EVE-3 (a splice variant of SPRED3; Wakioka et al. 2001, Kato et al. 2003, King et al. 2006). Common to both SPRED1 and SPRED2 are the EVH1 and CRD, separated by a highly divergent sequence encompassing the $\mathrm{c}$-Kit binding domain (KBD) to which the kinase domain of c-Kit receptor binds. SPRED3 lacks a functional KBD and consequently cannot bind to c-Kit. SPRED1 and 2 are tyrosine phosphorylated by stem cell factor, PDGF, and EGF and, like SPRY, SPRED can inhibit ERK phosphorylation stimulated by NGF, and FGF (Wakioka et al. 2001, Bundschu et al. 2007). It is presently unclear how SPRED proteins differ from SPRY proteins in exercising their respective inhibitory effects on the Ras/ERK pathway, however, studies have shown that, under certain conditions, both the EVH1 and the CRD are indispensable for this function (King et al. 2005). SPRED3 shows less inhibitory activity than SPRED1 and 2, suggesting that a functional KBD may also be required. Interestingly, the liver-restricted EVE3 contains a single EVH1 domain and, like SPRED, is capable of inhibiting Ras/ERK signaling (King et al. 2006). This emphasizes the relative importance of the EVH1 domain for SPRED proteins in their inhibitory function. As yet, no bona fide interacting partner for the EVH1 domain of SPRED has been identified, while a number of proteins bind to the common CRD of both SPRED and SPRY family members. Complicating matters further, both SPRED proteins can homodimerize and heterodimerize via the CRD (King et al. 2005), and the possibility remains that SPRED and SPRY proteins can heterodimerize via the same mechanism.

The major mode of action of the SPRY family of proteins in cell signaling remains elusive, and while interacting partners for SPRY are constantly unveiled, we are still at a loss to locate some 'universal rules' on how such complexes work in a physiological context. Here, rather than simply summarizing the present SPRY literature, we endeavor to surmise the likely function of the SPRY proteins, particularly SPRY2, using the relevant findings presently put forward in the SPRY field to support these assumptions and predictions. The review is primarily limited to assess the effect of proteins that have substantially been shown to bind directly to SPRY proteins or directly modify them.

\section{Common sequence motifs delineating SPRY functions}

For signaling proteins that lack a presently-recognized interaction domain, their function may be implied by identifying proteins that associate directly with them: the 'guilty-by-association' approach. This is usually matched with 
a detailed analysis of common sequence motifs within the family of proteins and, if such a motif has been conserved throughout evolution, it can be assumed that it is vital to the function of the proteins in which it is found. Such an analysis of the Drosophila and four mammalian SPRY proteins, combined with prediction and experimental evidence, aided in the identification and characterization of several conserved sequences, of which three are now well-defined (Fig. 1): 1) the canonical Casitas B-lineage lymphoma (c-Cbl) tyrosine kinase-binding (TKB) motif centered around a key tyrosine reside (Y55), 2) the serine-rich motif (SRM) and 3) the cysteine-rich domain (CRD; also referred to elsewhere as the SPR domain or the translocation domain).

\section{The canonical Cbl-TKB binding site}

Early experimental evidence indicated that SPRY2 was tyrosine phosphorylated upon stimulation and that the tyrosine residue at amino acid position 55 was the main target (Sasaki et al. 2001, Fong et al. 2003, Mason et al. 2004). Prior to this,
SPRY2 was observed to bind to the c-Cbl E3 ubiquitin ligase as a consequence of FGFR and EGFR activations (Wong et al. 2001) and it later emerged that this same Y55, when phosphorylated, was in the center of the canonical $\mathrm{Cbl}$ TKB motif: N-X-Y(p)-S/T-X-X-P (Lupher et al. 1997). The TKB domain is structurally an $\mathrm{SH} 2$ domain in association with a $4 \mathrm{H}$ domain and an EF-hand, and completes its unique binding capabilities through the interactions of these subdomains with the substrate protein (Meng et al. 1999). This motif was one of three known Cbl-TKB binding motifs, and proteins were classified into groups based on these derivative consensus sequences. Crystallographic evidence now suggests that $\mathrm{Cbl}$ in fact recognizes a less-radical binding motif, with $\mathrm{N}-\mathrm{X}-\mathrm{Y}(\mathrm{p})$ or $\mathrm{R}-\mathrm{Y}(\mathrm{p})$ essential for binding, and the other conserved residues required to enhance the binding affinity ( $\mathrm{Ng}$ et al. 2008). It is interesting to note that the targeted proteins are either receptor and non-RTK kinases (e.g. EGFR and Lck) or adaptor proteins such as the members of the APS family. Some of the binding proteins are Cbl-mediated ubiquitin targets (c-Met; Peschard et al. 2001)
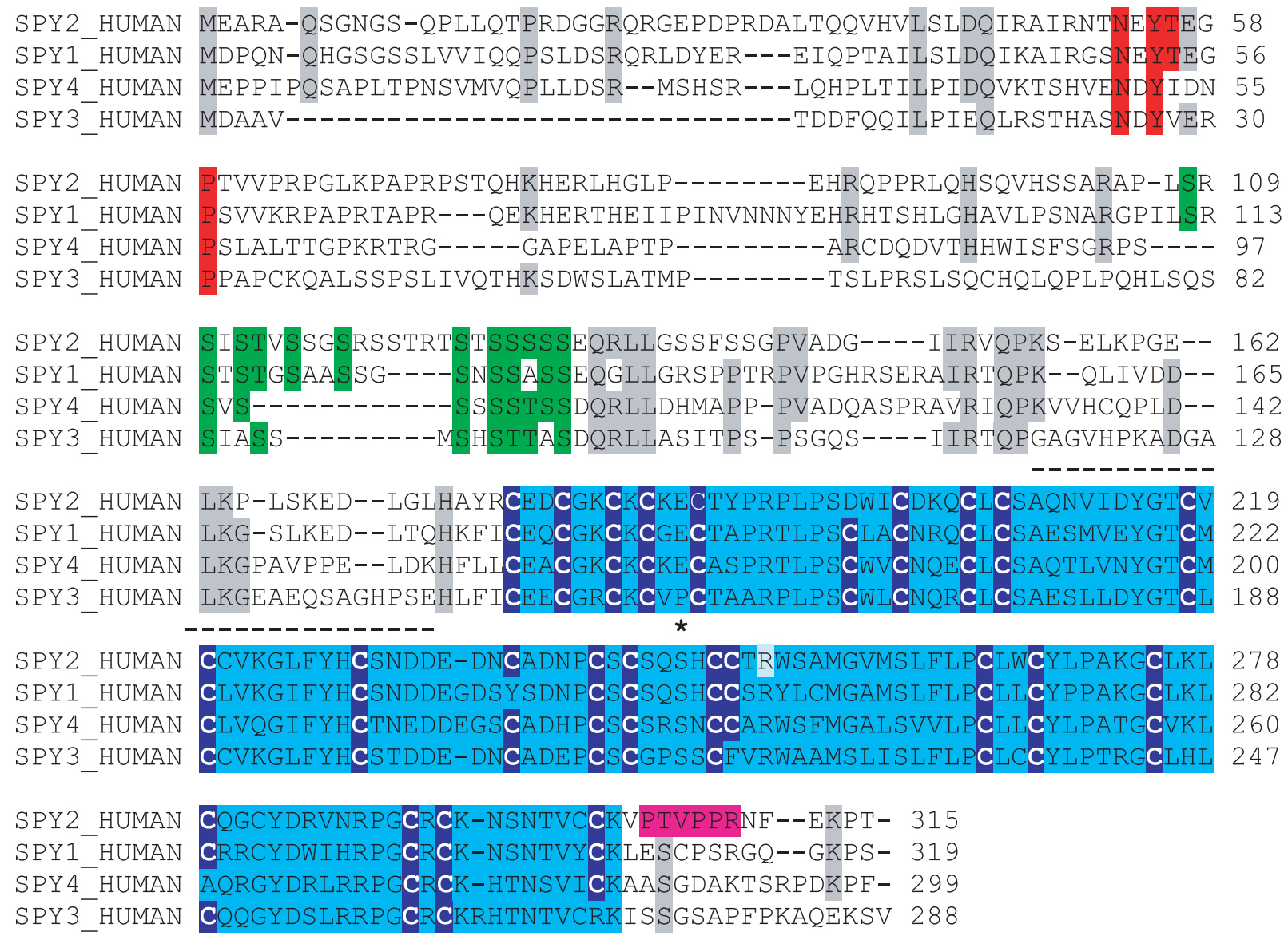

Figure 1 The conserved motifs/domains of Sprouty proteins. The three conserved sequences on Sprouty proteins are indicated: the cysteine-rich domain (shaded in light and dark blue), the serine-rich motif (conserved S/T residues are shaded in green), and residues from the canonical CbI TKB binding site (shaded in red). Several other sequences that are conserved without any assigned function are shaded in grey. The proline motif on Sprouty2 that binds $\mathrm{SH} 3$ domains is shaded in pink. 
Table 1 Cbl tyrosine kinase-binding (TKB)-binding motifs

\begin{tabular}{|c|c|}
\hline & \\
\hline & $\begin{array}{lll}-2 & 0 & +4\end{array}$ \\
\hline & \\
\hline & $\begin{array}{l}\text { NXYpSXXP } \\
\text { D T }\end{array}$ \\
\hline ZAP -70 & TLNSDGYPTPEPA \\
\hline P75NTR & KGDGNLYPSSLPL \\
\hline SRC/FYN & LIEDNEYPTARGQ \\
\hline CSF-1R & LLQPNNYPQFC \\
\hline SYK & TVSFNPYPEPELA \\
\hline------ & - - - - - - - - - \\
\hline mSPRY2 & IRNTNEYpTEGPT \\
\hline mSPRY1 & IRGSNEYPTEGPS \\
\hline mSPRY4 & SHVENDYPVDTPL \\
\hline dSPRY & ERLTNEYPVDTPL \\
\hline
\end{tabular}

The amino acid motifs on various proteins have been shown experimentally to bind to the TKB domain of Cbl protein. The consensus motif is shown on the top of the table with the phosphorylated tyrosine designated by convention as the zero reference point. The motifs in the top half of the table all come from tyrosine kinases and those in the bottom half from Sprouty family proteins. There are two other groups of TKB-binding motifs (not shown) that have derivative binding sequences.

while others apparently utilize $\mathrm{Cbl}$ as a docker protein (APS; Hu \& Hubbard 2005).

With respect to the original, 'ideal' canonical binding sequence, Table 1 shows that four proteins - ZAP70, p75NTR, SPRY1 and SPRY2 - each retain the four conserved amino acids. This being the 'ideal' motif is reflected in the strength of binding ascertained for SPRY2 over other TKB binding peptides that dispensed with one or more of the conserved residues ( $\mathrm{Ng}$ et al. 2008). The crystallographic results demonstrated that the phosphorylated tyrosine and the proline in the +4 position (relative to the central tyrosine) occupies a positively-charged pocket and a shallow hydrophobic cleft on the TKB domain of Cbl respectively (Fig. 2). However, this proline residue was deemed non-essential for binding; rather it contributes to the overall affinity of the phosphorylated peptide for the TKB domain. Similarly, the conserved serine/threonine residue at the +1 position is also employed only to increase the binding affinity, as verified by SPRY2's higher affinity for Cbl over SPRY4 which lacks this residue. Interestingly, there is recent evidence to suggest that this threonine residue in SPRY is phosphorylated in cultured cells (Sweet et al. 2008); how this phosphorylation event affects the phosphorylation of the conserved tyrosine or the binding affinity of SPRY to Cbl will be interesting to assess.

Unlike the APS protein that employs c-Cbl as an adapter protein, our group and others have demonstrated that, in over-expression systems, the binding of SPRY2 to c-Cbl results in the ubiquitination and subsequent destruction of SPRY2 in the endosomal compartment, and it was interpreted that the level of SPRY2 in cells is controlled in this manner (Hall et al. 2003, Rubin et al. 2003). In other experiments, when SPRY2 was over-expressed in cultured cells, EGF stimulation failed to cause the Cbl-directed downregulation of the EGFR and instead led to sustained ERK signaling (Wong et al. 2002b, Hall et al. 2003, Rubin et al. 2003). At the time, this was presumed to be the result of the selective sequestration of $\mathrm{Cbl}$ by the high levels of SPRY2, and our later work comparing the binding affinities of SPRY2 and EGFR peptides with the TKB domain suggests that this could theoretically be achieved ( $\mathrm{Ng}$ et al. 2008).

While this possibility stands, in our reasoning, it seems unlikely that SPRY2 would have such high affinity for $\mathrm{c}-\mathrm{Cbl}$ simply to sequester it from acting in other systems, particularly since $\mathrm{Cbl}$ tightly controls itself in order to ensure that excessive signaling does not ensue from RTKs, such as FGFR and possibly EGFR. Therefore, we envisage that the binding of c-Cbl to SPRY proteins has one of the three likely physiological outcomes: 1) SPRY is a Cbl-directed ubiquitin
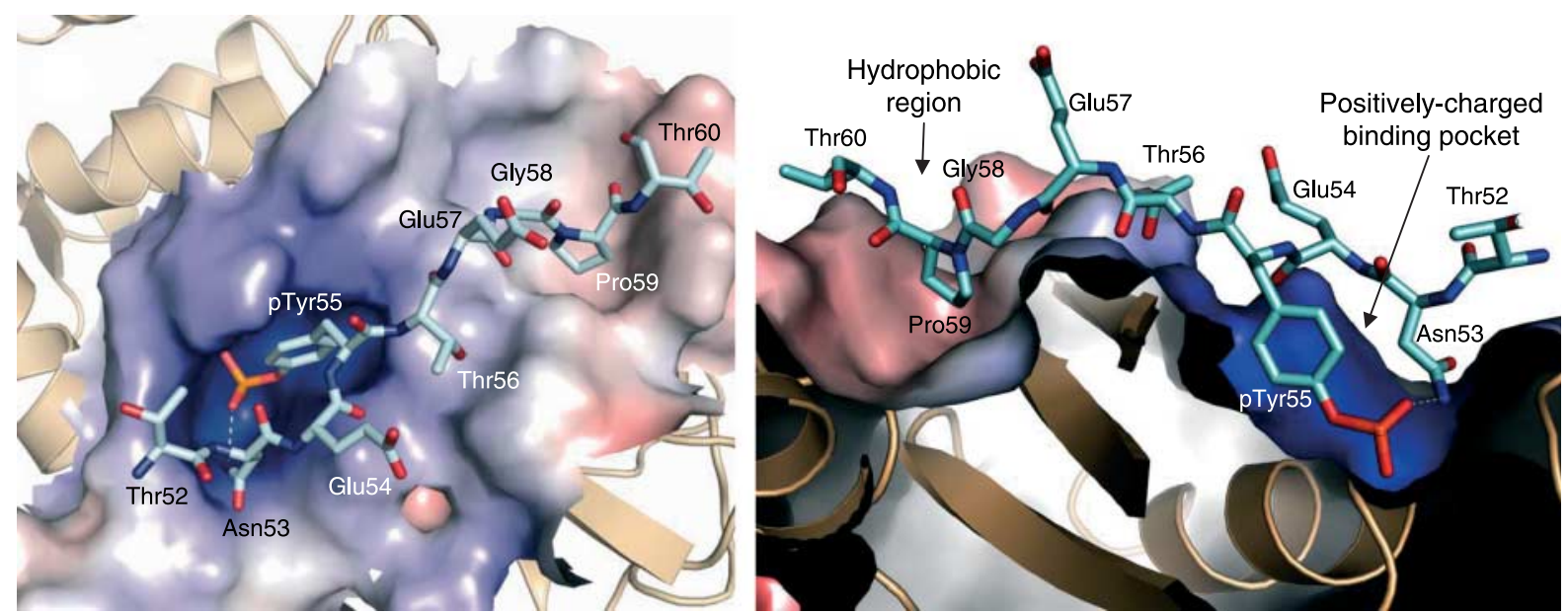

Figure 2 Binding of SPRY2 to the c-CbI TKB domain. Crystal structure and electrostatic surface representations of the TKB complexed with SPRY $2^{49-61}$ from two perspectives: looking down (left) and cut-away (right). The longer arrow indicates the position of the phosphorylated tyrosine in a positively-charged pocket and the shorter arrow indicates the proline residue $(+4)$ in a shallow hydrophobic cleft. Hydrogen bonds between the TKB domain and the peptide are shown as grey dotted lines (derived from $\mathrm{Ng}$ et al. 2008). 
target, 2) SPRY targets Cbl to other proteins for ubiquitination, and/or 3) SPRY functions as an adaptor protein for $\mathrm{Cbl}$, utilizing its scaffolding but not its ubiquitin E3 ligase function. In the first scenario, SPRY proteins would be the front-line targets for $\mathrm{c}-\mathrm{Cbl}$ directed ubiquitination as their presence in the cell is required only in a time-restricted manner. Furthermore, in our unpublished and published work, it has been noted that high affinity binding does not necessarily correlate with ubiquitination: APS binds Cbl strongly but is not a substrate for ubiquitination (Hu \& Hubbard 2005) and the converse applies to c-Met (Peschard et al. 2001). However, it does appear somewhat counterintuitive that one downregulator of the ERK pathway itself is targeted for destruction by another down-regulator of the same pathway.

Sprouty proteins may also act as facilitators, targeting $\mathrm{Cbl}$ to other proteins to which $\mathrm{Cbl}$ does not directly bind, resulting in the ubiquitination of these targeted proteins. A similar example of targeting of $\mathrm{Cbl}$ exists between $\mathrm{c}-\mathrm{Cbl}$ and the adaptor protein GRB2 in FGF signaling (Wong et al. 2002a). GRB2 mediates the binding of $\mathrm{c}-\mathrm{Cbl}$ to the FRS2/FGFR complex following ligand stimulation and results in the ubiquitination and down-regulation of both FRS2 and receptor. More recently, the ubiquitination of the $\beta$-subunit of the interleukin-6 receptor (also known as gp130) was shown to be mediated by $\mathrm{c}-\mathrm{Cbl}$ via its association with SHP2 (listed as PTPN11 in the Hugo Database) acting in its capacity as an adaptor protein (Tanaka et al. 2008). In either case, by binding to both $\mathrm{Cbl}$ and SHP2, SPRY2 may be a necessary link to bring $\mathrm{Cbl}$ into contact with its substrates via SHP2.

The third potential role for SPRY with respect to its interaction with $\mathrm{c}-\mathrm{Cbl}$ is as an adaptor protein. SPRY could then make functional use of Cbl's wide range of associate proteins as opposed to its ubiquitin E3 ligase ability (Schmidt \& Dikic 2005, Thien \& Langdon 2005). There is precedence for this in insulin signaling, where $\mathrm{Cbl}$ is targeted to the insulin receptor via the APS adaptor protein. Neither APS nor the insulin receptor is ubiquitinated by $\mathrm{Cbl}$ in the resulting complex; rather $\mathrm{Cbl}$ facilitates GLUT4 translocation within the cell (Hu \& Hubbard 2005).

While a case for $\mathrm{Cbl}$ can be put forward, another ubiquitin E3 ligase also binds to the N-terminal of SPRY2. In a yeast two-hybrid screen, Nadeau et al. (2006) identified human seven in absentia homolog 2 (SIAH2) as a SPRY2 interacting protein. The N-terminal half of Spry2 was demonstrated to interact with the ring finger domain of SIAH2 in a tyrosine phosphorylation-independent manner. Over-expressed SIAH2 initiated the ubiquitination and subsequent degradation of SPRY2, SPRY1, and to a lesser degree SPRY4. It is possible that much of the predictions and speculation made for $\mathrm{Cbl}$ in the preceding text could also apply to SIAH2.

In addition to acting as a binding site for Cbl, the Y55 residue has also been shown to be essential for the binding of the protein phosphatase 2A (PP2A; Lao et al. 2007). DaSilva et al. (2006) demonstrated that the degree of Y55 phosphorylation and the stability of Spry2 were affected by the serine/threonine phosphorylation status of certain residues in the SRM of the protein. In support of this, Lao et al. (2007) provided evidence that, under stimulated conditions, SPRY2 requires the dephosphorylation of certain serine residues within the SRM to change its tertiary structure and permit GRB2 binding to a canonical SH3 binding motif on the C-terminus. The trimeric PP2A phosphatase was shown to be responsible for this dephosphorylation, binding to a motif on SPRY2 surrounding the Y55 residue via the scaffolding A subunit (Lao et al. 2007). It is presently not known whether phosphorylation of Y55 favors this interaction, and similarly, the necessity of a phosphorylation event on the +1 threonine is also yet to be established.

\section{Regulating the Y55 residue}

The accumulated evidence clearly indicates that the phosphorylation status of the Y55 residue plays a major role in the physiological function of SPRY, particularly for its activity as an inhibitor of the Ras/ERK/MAPK signaling cascade. The phosphorylation status of Y55 is dependent on the relative activity of the Y55 kinase(s) and Y55 phosphatase(s) with which it interacts. Present evidence indicates that c-Src (or a related kinase) is the tyrosine kinase responsible for phosphorylating the Y55 residue (Li et al. 2004). Interestingly, the c-Src autophosphorylation site, E-NE-Y-T (Bjorge et al. 2000), is almost identical to the motif surrounding the Y55 residue in SPRY1 and 2, with SPRY3, SPRY 4 and $d$ Spry lacking the threonine in the +1 position. It has been noted in several labs that while SPRY1 and 2 become strongly phosphorylated upon FGFR stimulation, the phosphorylation of SPRY4 is relatively weak. These differing degrees of phosphorylation parallel the relative degrees of binding of SPRY proteins to c-Cbl both in vivo and in vitro, with SPRY4 showing weak binding compared with SPRY2 (Fong et al. 2003, Ng et al. 2008).

The activation of the Ras/ERK pathway downstream of a number of RTKs has been demonstrated to require the phosphatase activity of SHP2 (Araki et al. 2003). In fibroblasts, SHP2 undergoes phosphorylation in two C-terminal tyrosyl residues in response to FGF and PDGF but not other growth factors, such as EGF or insulin-like growth factor. Downstream of the FGFR, SHP2 binds to two phosphorylated tyrosines on FRS2, to which the SOS-GRB2 complex is also linked via GRB2, and thus facilitates the activation of membrane-located Ras and downstream ERK signaling (Hadari et al. 1998). The substrate(s) for SHP2 requiring dephosphorylation in this context has been sought for some time. It was reasoned that SPRY proteins, being tyrosine phosphorylation-dependent negative regulators of the ERK pathway, would enable activation of the pathway when functionally inactivated by the dephosphorylation of Y55. Several groups have provided evidence that SPRY1 and SPRY2 bind to SHP2, and Hanafusa et al. (2004) demonstrated that the expression of an activating SHP2 mutant (Shp2E76A) leads to enhanced dephosphorylation of 
over-expressed Spry 1 and Spry 2 which, in turn, resulted in the dissociation of the SPRY2-GRB2 complex and prolonged ERK activation. Jarvis et al. (2006) demonstrated that SHP2 binds to over-expressed SPRY1 resulting in the dephosphorylation of the Y55 equivalent (Y53) as well as Y89. Chan et al. (2008) later pointed out, however, that a more stringent proof is required for SPRY1 or 2 to be deemed to be a substrate of SHP2 as mice deficient in SPRY1, SPRY2 or SPRY4 fails to display a phenotype synonymous with Noonan syndrome (NS), a relatively common congenital genetic condition distinguished by heart malformation, short stature, learning problems, indentation of the chest, impaired blood clotting, and a characteristic configuration of facial features (Tartaglia et al. 2001). One study reports that approximately half of the patient cohort with NS carried a PTPN11 mutation, which encodes the protein tyrosine phosphatase SHP2 (Tartaglia et al. 2001). Thus, it would be expected that mice deficient in SPRY would display similar phenotypes to NS if SHP2 were a substrate. Chan et al. (2008) suggest that the SPRED proteins are more likely substrate candidates, as germ line loss-of-function mutations in SPRED1 cause a variant NS-like syndrome.

\section{The serine-rich motif}

An alignment of the mammalian SPRY proteins (Table 2) indicates that there is a strong conservation and concentration of mainly serine residues and a few threonine residues between amino acid residues 107-132 (on SPRY2), otherwise known as the SRM. It is noteworthy that this motif is only weakly conserved in $d S p r y$. A preliminary examination of the sequence indicates that the conserved serine residues occur $\mathrm{N}$-terminal to a conserved acidic residue (green), which is characteristic of consensus phosphorylation motifs for the kinases CK1 (S/T-X-X-S), CK2 (S/T-X-X-D/E), and GSK3 (S/T-X-X-X-pS; Ubersax \& Ferrell 2007).

SPRY proteins were originally shown to be predominantly phosphorylated on serine residues by Impagnatiello et al. (2001). It was noted that SPRY2 migrated as two major bands on SDS-PAGE gels and that the slower migrating band could be eliminated by alkaline phosphatase treatment.

Table 2 Serine-rich motif

\begin{tabular}{|c|c|c|}
\hline \multicolumn{3}{|c|}{ * $\quad$ * } \\
\hline & + & \\
\hline 108 & SRSISTVSSGSRSSTRTSTSSSSSE & 132 \\
\hline 112 & SRSTSTGSAASSG--- - SNSSASSE & 132 \\
\hline 98 & SVS ----------- SSSSTSSD & 108 \\
\hline 81 & QSSIASS--- - - - MSHSTTASD & 96 \\
\hline
\end{tabular}

An alignment of the conserved serine-rich motif of Sprouty family proteins. Serines (or threonines) that are conserved in the majority of the proteins are in red. A conserved acidic residue (E or D) that is likely to be part of a kinase recognition motif is in green. The + above the SPRY2 sequence indicates serine residues that were shown to be phosphorylated by Mnk (DaSilva et al. 2006) while the $*$ indicates the serine residues on SPRY2 that were dephosphorylated by PP2A on FGFR activation (Lao et al. 2007).
Our laboratory identified that the characteristic, differential migration of SPRY2 on SDS-PAGE gels was indicative of the phosphorylation of certain serine/threonine residues on SPRY2, and not the overall phosphorylation status of the protein. (It should be noted here that SPRY1 shows a modest separation of isoforms, whereas SPRY4 is similar to SPRY2). It is hypothesized that this conserved serine motif might be part of or controls a critical hinge region on the SPRY proteins; it appears that dephosphorylation within the motif is the 'on' switch and phosphorylation, the 'off' switch. Mass spectrophotometric analysis of FGF-stimulated cells indicated that Ser115 and Ser118 at least were dephosphorylated upon stimulation whereas there was a net increase in phosphorylation on other sites (Lao et al. 2007).

There have been several kinases shown to bind to SPRY proteins and cause their altered migration or 'band shifting' on SDS-PAGE gels: isoforms of CK1, CK2, and TESK1 (Chandramouli et al. 2008, unpublished data). TESK1 causes band shifting of SPRY2 when transfected into cells, however, there is no apparent consensus phosphorylation motif for this kinase in the SRM; band shifting may be arising from unidentified phosphorylation sequences, indirectly through another kinase(s) or perhaps even through directed serine/ threonine phosphatase activity. MNK1 has also been shown to cause band shifting of SPRY2 but it was not confirmed to be binding to SPRY2. DaSilva et al. (2006) demonstrated that this kinase phosphorylated Ser112 and Ser121 within the SRM of SPRY2, resulting in an increase in the stability of the protein in comparison with the unphosphorylated state. This is probably further evidence that the phosphorylation status of residues within the SRM affects the tertiary structure of SPRY2. A corollary of dephosphorylation of these two serine residues is an increase in the tyrosine phosphorylation of Y55 and its interaction with other binding proteins.

While a handful of kinases can phosphorylate serine residues within the SRM, the phosphatase that appears responsible for dephosphorylating these residues is PP2A (Lao et al. 2007). Following FGFR activation, PP2A was activated and shown to be responsible for dephosphorylating Ser115 and Ser118 in a restricted identification of specific substrates. The apparent competitive binding of $\mathrm{Cbl}$ and PP2A - the former apparently responsible for degrading SPRY2 while the latter activates the ERK phosphorylation inhibitory action of SPRY2 - led us to propose a model, where incoming signals essentially provide an 'on' and 'off' switch for SPRY2 function (Fig. 3).

\section{The cysteine-rich domain}

A number of well-characterized protein-interaction domains feature a grouping of cysteine residues. This eclectic group of proteins that contain CRD has various functions from membrane-targeting to chelating metal ions. For example, the CRD of Raf1 (residues 139-184), consisting of two zinc finger motifs analogous to the phorbol ester binding-C1 


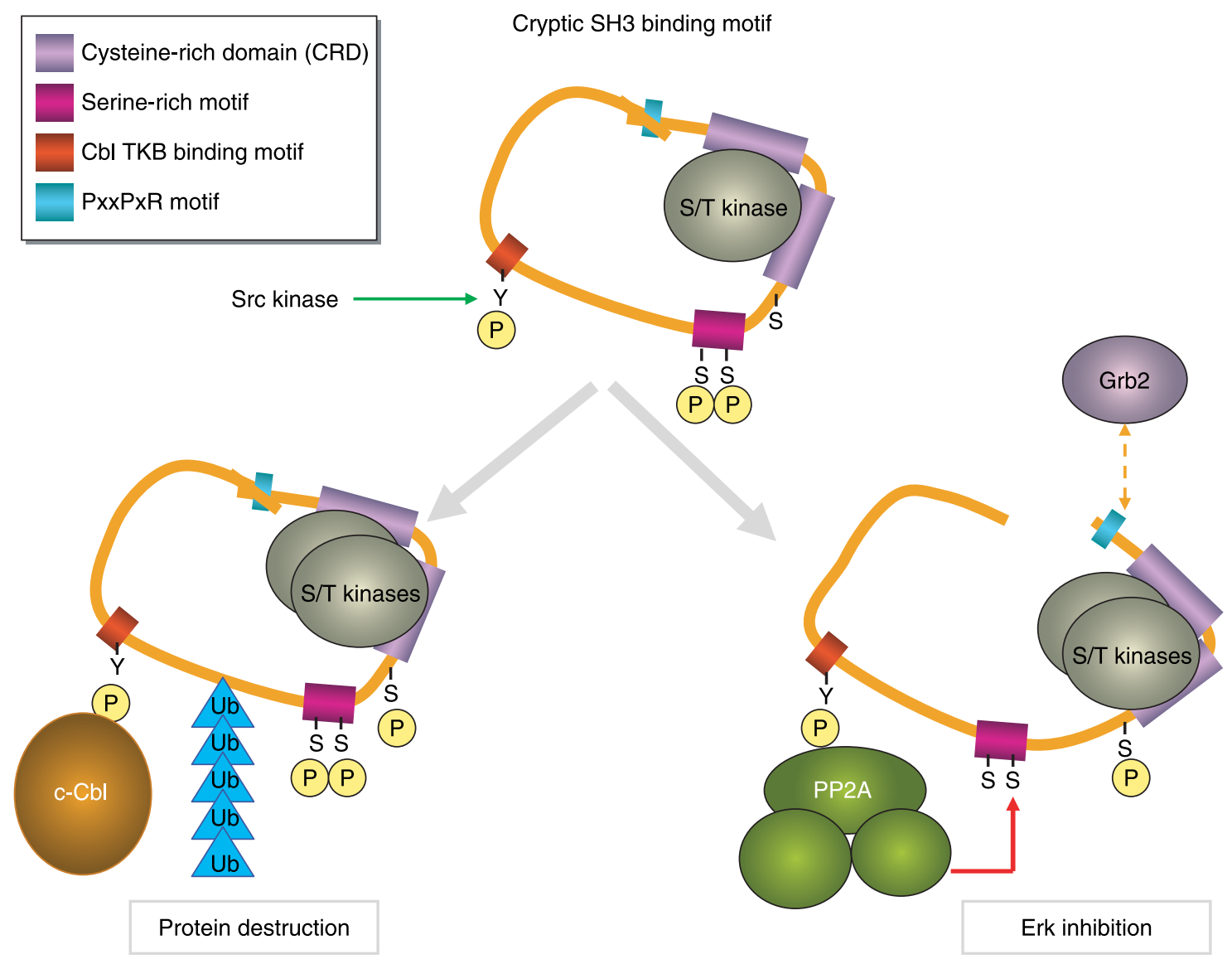

Figure 3 Activation versus degradation of SPRY2. A stylized diagram to illustrate the dynamic nature of Sprouty's fate following tyrosine phosphorylation of Y55. The TKB domain of c-Cbl binds the consensus motif and directs the ubiquitination and subsequent destruction of Sprouty2. PP2A subunits compete for binding with c-Cbl and form a platform for the selective dephosphorylation of serine residues within the serine-rich motif. During this process, Sprouty2 undergoes a change in tertiary structure that exposes the otherwise cryptic SH3-binding motif on the C-terminus enabling interaction with GRB2 and other SH3-containing proteins. In this 'activated' form, Sprouty2 is able to inhibit the phosphorylation (and activation) of ERK.

domain of protein kinase $\mathrm{C}$, binds to Ras with relatively low affinity, and works in concert with the Ras binding domain to fully augment the Ras/Raf functional association and facilitate the downstream signaling (Mott et al. 1996, Okada et al. 1999). By contrast, the CRDs of other proteins play varying roles: the $\mathrm{CRD}$ of the calcium-sensing receptor (hCaR) is necessary for signal transmission from the $\mathrm{N}$-terminal venus-flytrap domain to the seven-transmembrane domain ( $\mathrm{Hu}$ et al. 2001, Tan et al. 2003); the mannose receptor uses the CRD as a carbohydrate recognition motif (Fiete et al. 1998); the CRD from a rubella virus non-structural protein is essential for viral protease activity and virus replication (Zhou et al. 2009); the scavenger receptor MARCO utilizes the CRD for ligand recognition (Ojalo et al. 2007); and the disintegrinlike/CRD of ADAM12 functions as a cell adhesion domain (Zhao et al. 2004).

Unsurprisingly, the CRD in the various SPRY proteins shows no functional similarity to any of those previously described. Intuitively, it might then be assumed that this shared domain is central to the mode of inhibition of the SPRY and SPRED families with respect to ERK inhibition, but evidence for this hypothesis is presently lacking. The CRD of SPRY was shown to mediate both homo- and heterodimerization between its family members (Ozaki et al. 2005). The CRD was also shown to influence the function of SPRY by localizing them to the cell membrane, both in Drosophila and in vertebrates (Casci et al. 1999, Lim et al. 2000). Membrane targeting was attributed to palmitoylation of the unusually large number of cysteine residues in this region (Impagnatiello et al. 2001), and its binding to the lipid PtdIns(4,5)P2, predominantly found on the plasma membrane of activated cells (Lim et al. 2002). The functional importance of the latter observation was validated by the finding that a mutant of SPRY2 (R242D) that was defective in binding PtdIns(4,5)P2, not only failed to translocate to the membrane, but also lost its ERK inhibitory capacity. The CRD domain of the SPRED family of proteins share these features including membrane translocation, binding to 
PtdIns(4,5)P2, and the loss of these functions when the implicated arginine residue is mutated, and thus it has been speculated that the function of the CRD in SPRED also lay in directing the localization of the protein.

However, a second role for this domain in protein-protein interactions later came to light when binding to Raf1 was reported for both SPRY and SPRED by virtue of the CRDs (Sasaki et al. 2003). Later, two independent yeast two-hybrid screens for two kinases demonstrated an interaction with the SPRY family members via the CRD: the testicular protein kinase (TESK) and the dual-specificity tyrosine-phosphorylation-regulated protein kinase (DYRK). Leeksma et al. (2002) showed that the CRD of SPRY4 binds directly to TESK1, a serine/threonine kinase related to LIM kinases, and later demonstrated that the complex inhibits the kinase activity of TESK1, suppressing cofilin phosphorylation and the subsequent formation of stress fibers and focal adhesions via the C-terminal 100 amino acids of TESK1 (that are absent in the non-binding TESK2; Tsumura et al. 2005). Chandramouli et al. (2008) later showed that all SPRY and SPRED proteins bind to TESK1, but not TESK2, via their respective CRDs. Like the case of SPRY4, SPRY2 binding to TESK1 suppressed cofilin phosphorylation, and also suppressed the inhibitory action of SPRY2; the latter is likely caused by sequestering SPRY2 away from interacting partners such as the PP2A-A and C subunits. The TESK1-SPRY2 interaction caused an increase in the slower migrating band of SPRY on SDS-PAGE gels, indicating that the kinase affects the phosphorylation of serine (or threonine) residues within the SRM of SPRY2; whether this is direct phosphorylation by the kinase or via indirect mechanisms is yet to be ascertained.

A more recent yeast two-hybrid screen using the dualspecificity tyrosine phosphorylation-regulated kinase (DYRK1A) as bait identified SPRY2 as a binding partner. Aranda et al. (2008) demonstrated that DYRK1A interacts with and regulates the phosphorylation status of SPRY2, and identified Thr75 on SPRY2 as a DYRK1A phosphorylation site in vivo and in vitro. The site appeared to be functional in that its mutation modestly enhanced the repressive function of SPRY2 in FGF-induced ERK signaling, and the two proteins were shown to co-localize in several structures in the mouse brain.

While there is commonality in the binding of the CRD of SPRED and SPRY, there is also variation. For instance, it has

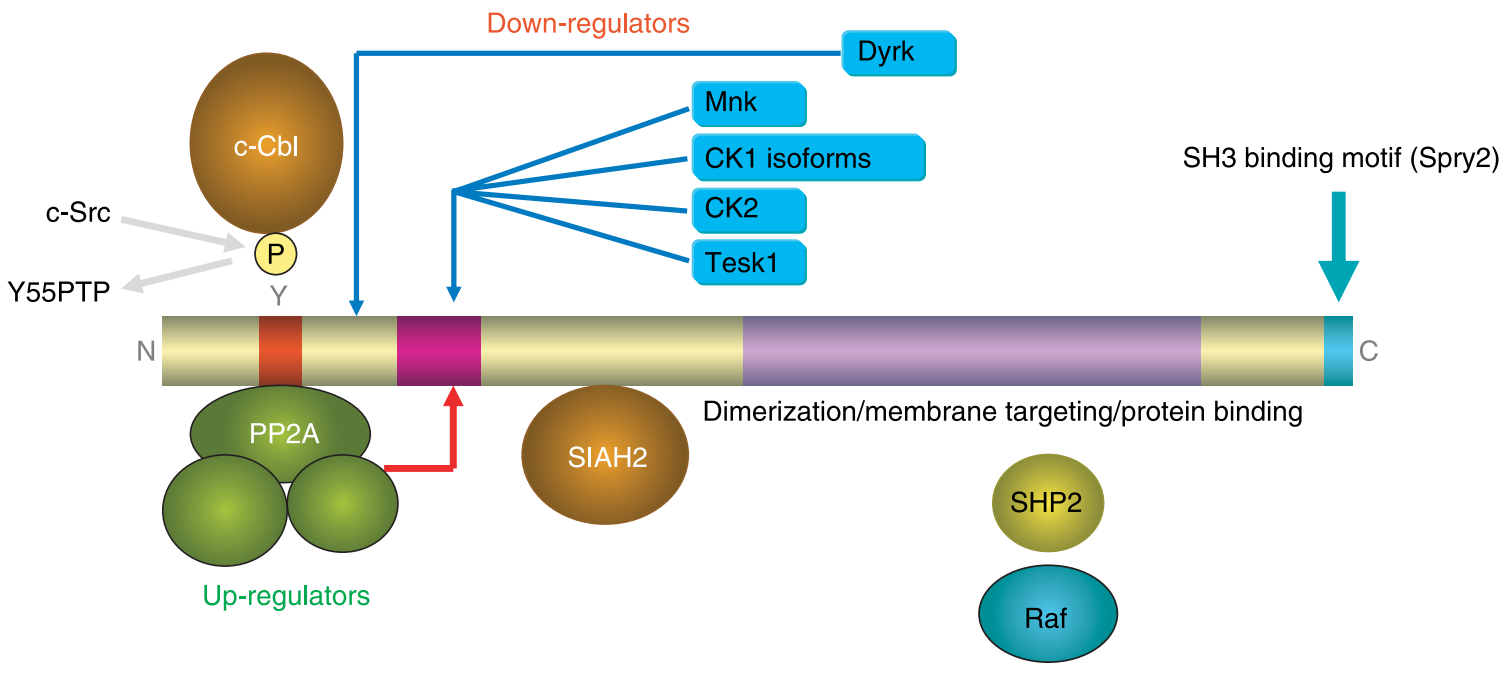

Cysteine-rich domain (CRD)

Serine-rich motif

Cbl TKB binding motif

PxxPxR motif

Figure 4 The covalent modifiers of SPRY proteins. Sprouty's presentation within the cell and its likely function are controlled by covalent modification: predominantly phosphorylation and ubiquitination. The location of the binding and targeting for the Ser/Thr kinases is indicated. The binding location of MNK has not been specified, while the target of DYRK appears to be on the $\mathrm{N}$-terminus, outside the serine-rich motif (SRM). Two ubiquitin E3 ligases, Cbl, and SIAH2, bind to the N-terminus of SPRY, whereas the SHP2 tyrosine phosphatase and Raf kinase bind to the cysteine-rich domain (CRD). Although capable of modifying targets on Sprouty, there is presently no firm evidence that SHP2 and Raf do so; SHP2 likely has a scaffolding function. It presently appears that covalent modifications that decide the activity and fate of SPRY occur on the N-terminus while the majority of binding occurs on the CRD. 
been observed that although binding of Raf1 is mediated by the CRD of SPRED1, the binding of SPRED2 and most of the SPRY isoforms to Raf1 is minimal (our unpublished data). In addition, replacing the CRD of the relatively weak inhibitor SPRED3 with that of SPRED1 results in the chimera inhibiting ERK phosphorylation as strongly as SPRED1 itself (Kato et al. 2003). Furthermore, there is an apparently functional variation among the CRDs of the SPRED family; King et al. (2005) provided evidence that while the CRD mediates heterodimer formation, the CRD of SPRED2 is required for ERK inhibition, whereas the equivalent domain of SPRED1 is not, indicating that the two isoforms use different mechanisms for the inhibition of the ERK pathway. A recent report implicated the CRD of SPRY in mediating its binding to Caveolin1 (Cabrita et al. 2006), another protein that also binds to SPRED (Nonami et al. 2005) and, although the region for mediating this binding was not demonstrated, they revealed that various SPRY2 mutants (including the CRD mutation at R242D) failed to bind to caveolin and concomitantly showed a compromised ability to downregulate ERK phosphorylation.

It is apparent that a substantial fraction of the CRDinteracting proteins are kinases. Our laboratory has also recently characterized an interaction with kinases from the PKC family that associate with the CRD of Spry proteins in conjunction with other regions of the protein (Chow et al. 2009). The interaction occurs upon stimulation and results in the inhibition of the downstream substrate PKD1 that was also involved in a trimeric complex with SPRY2 and $\mathrm{PKC} \delta$. In the early phase of this study, another

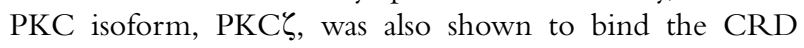
domain of all the SPRY isoforms (our unpublished data).
A summary of the binding location and target sites of various enzymes that covalently modify SPRY proteins is shown in Fig. 4.

\section{Sprouty function: the unanswered questions}

Integrating the present information, it emerges that SPRY is fulfilling a role as a consummate integrator. It is stringently controlled both at the expression level and by a number of covalent modifications. The expression occurs as a result of the stimulation of at least one of the pathways that it feeds back on. Once expressed, evidence suggests that SPRY is in an inactive/inaccessible conformation and requires the appropriate dephosphorylation of key residues within a specialized hinge region for its activation.

The evidence that SPRY proteins are targets for ubiquitination and subsequent destruction by at least two ubiquitin E3 ligases (Cbl and SIAH2) indicates that it is in the cell's interest to limit the duration of the presence of SPRY. There also appears to be some form of competition between activators (PP2A) and downregulators (c-Cbl). Being responsive to a number of kinases, and the pathways they represent, would enable SPRY proteins to sense the intracellular environment via the activation status of the associating kinases. If the various kinases and phosphatases described above are altering the state of readiness of SPRY proteins, in particular SPRY2, a major question emerges: what proteins are being brought into a complex by SPRY to specify its major function?

As described above, there is strong evidence that $\mathrm{c}-\mathrm{Cbl}$ may be one of these key proteins. If we assume that SPRY1 or 2 is acting as an adaptor rather than being a substrate for $\mathrm{c}-\mathrm{Cbl}$
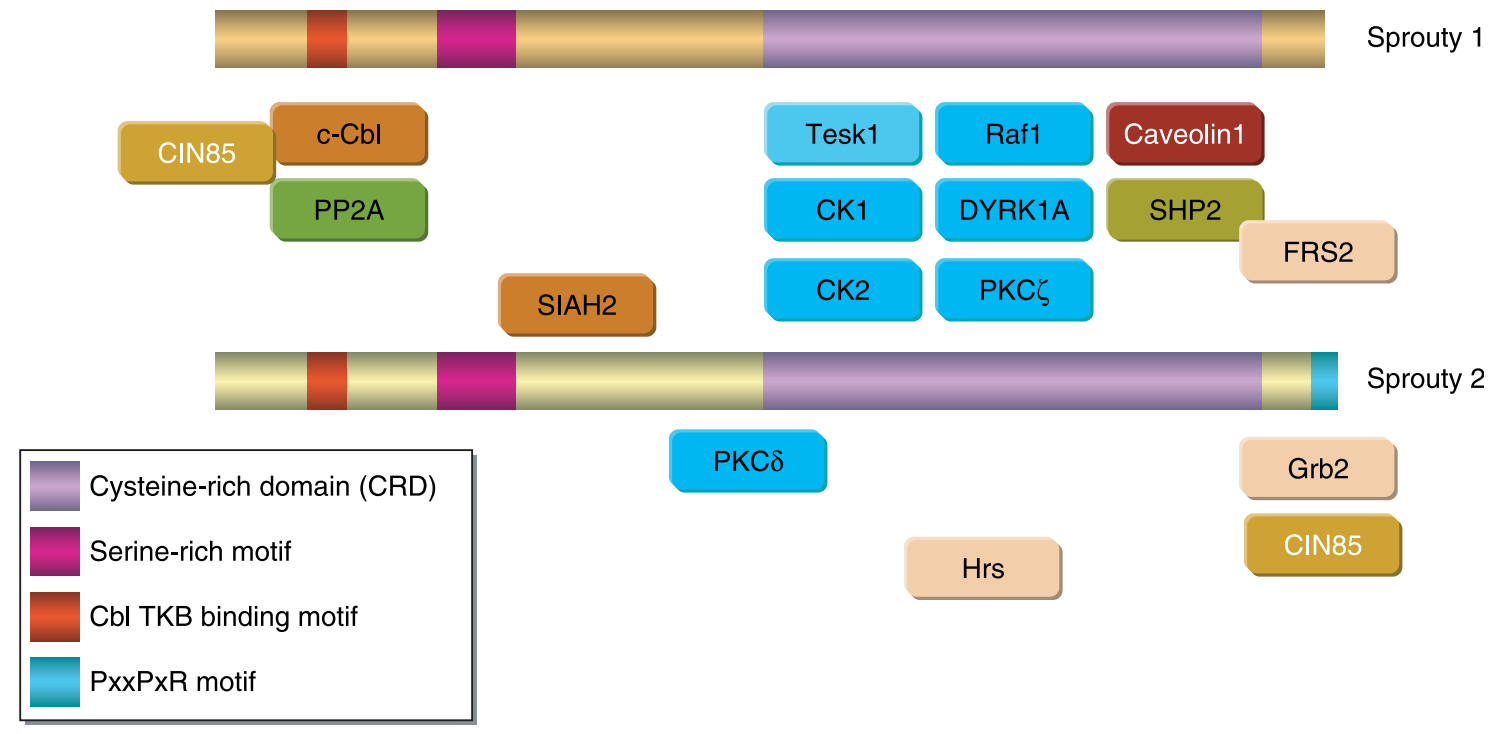

Figure 5 Sprouty binding proteins. A summary of the proteins that have been shown to bind to Sprouty 1 and 2 and the site to which they bind. The proteins depicted between the Sprouty proteins are common to both Sprouty 1 and 2, whereas those on the outside are specific to the adjacent binding partner. Kinases are in aqua, phosphatases in green, and Ubiquitin E3 ligases in pink and other proteins in shades of brown. PKC $\delta$ binds to several sites, and this is reflected in its ambiguous location. 
mediated ubiquitination, such as APS in insulin-mediated signaling, it is possible that these SPRY proteins bring $\mathrm{c}-\mathrm{Cbl}$ into close proximity of potential ubiquitination targets. Wong et al. (2002a) provided evidence that GRB2 recruits the $\mathrm{c}-\mathrm{Cbl}$ E3 ligase and its associated ubiquitination machinery into a complex with both FRS2 and FGFR1 to silence the activity of both signaling proteins. Recently, Tanaka et al. (2008) demonstrated that the tyrosine phosphatase SHP2, acting in its adaptor/docker role, associated with $\mathrm{c}-\mathrm{Cbl}$ and the gp130 cytokine co-receptor and this resulted in the ubiqutination and downregulation of the latter protein. From these data, it may be reasoned that $\mathrm{Cbl}$ could be targeted towards any protein to which GRB2 or SHP2 binds via their respective $\mathrm{SH} 2$ domain. It is notable that SPRY2 has been shown to bind to both FRS2 and SHP2 without GRB2 mediation and in both the above-mentioned cases SPRY1 or 2 could be the protein that complexes c-Cbl with FRS2 (and FGFR1) and SHP2 and hence gp130. Such connections would only involve SPRY 1 and 2 as the other SPRY proteins do not bind well to the TKB targeting sequence of Cbl. If this hypothesis was true, it would mean that each of the SPRY proteins would have distinctly different coordination tasks albeit with common modes of control.

There is also evidence that SPRY proteins are involved in the process of endocytosis of receptors; the association of SPRY2 with CIN85 (Haglund et al. 2005) and with Hrs (Kim et al. 2007). Direct binding in the latter case was not ascertained but it was demonstrated that hSPRY2 interferes with the ordered progression of ERK signaling from the early to late endosomes. This type of inhibition was similar in effect to that observed with Sef, which resides in the Golgi apparatus and inhibits MEK/ERK signaling specifically in this location (Torii et al. 2004). It was indicated that the interaction with Hrs offered an explanation for the observation that, contrary to expectations, SPRY2 up-regulated ERK signaling with EGF stimulation when genetic evidence indicated that SPRY proteins were downregulators of signaling (Kim et al. 2007). It could be postulated that SPRY2, CIN85 and Hrs cooperate in a complex to enhance the downregulation of certain RTKs. This aspect of SPRY function appears to warrant deeper investigation.

\section{Summary}

Presently, it appears that SPRY (and SPRED) proteins are integrators and sensors of cell signaling; some aspects of their function are relatively well-established and some questions are still to be determined:

1) The SPRY proteins have conserved the $\mathrm{c}-\mathrm{Cbl} \mathrm{TKB}$ binding site implicating $\mathrm{Cbl}$ as an important binding partner.

2) The SPRY proteins have a relatively well-conserved serine-rich domain that appears to have a function in the stability of the three-dimensional disposition of the protein by functioning as a structural 'hinge'.
3) SPRY2 has a canonical SH3 domain interacting prolinerich sequence. This sequence may be expected to interact with a number of SH3-containing proteins beyond GRB2.

4) The CRD remains enigmatic with evidence that it is involved in cellular location, dimer formation, and the binding of the majority of interacting proteins. While this domain is well-conserved, there is enough sequence variation to enable a degree of discrimination in binding among each of the SPRY and SPRED isoforms.

We are left with several major questions that will need to be addressed in order to fully comprehend the function of SPRY proteins in a broader context: 1) are SPRY proteins the major targets for Cbl-directed ubiquitination? If they are, then this suggests that it is desirable to confine the activation of SPRY to a defined temporal window. This is closely linked to the second question: 2) does SPRY direct $\mathrm{c}-\mathrm{Cbl}$ to an extended range of potential substrates for ubiquitination and downregulation? Presently, Cbl-directed ubiquitination is believed to be directed by $\mathrm{Cbl-TKB}$ motifs on proteins that require phosphorylation on a motif-central tyrosine residue. The SPRY-directed Cbl could extend targeting to other SPRYbinding proteins or even those associated with these proteins. There are no SPRY proteins in C. elegans, one in Drosophila and four in mammals, and the mechanisms of SPRY action are gradually being linked to signaling molecules that are derived from other signaling pathways, thus raising the question: 3) are SPRY proteins involved in signaling pathways other than the Ras/ERK pathway? There is strong genetic evidence that SPRY is involved in organogenesis, and it could be assumed that multiple isoforms of SPRY arose in higher mammals to enable specific functions in our more complex architecture, as compared to C. elegans and Drosophilia. It would be reasonable to expect SPRY to be involved in other signaling pathways given the nature of the associated proteins.

The assortment of protein types that are interacting with the SPRY proteins shows a number of kinases, two ubiquitin E3 ligases and several phosphatases (summarized in Fig. 5). There is a recent realization that ubiquitin addition and removal in its various combinations is likely to play a similar role to phosphorylation in cell signaling. The two pathways indulge in cross-talk, cooperation, and augmentation, and it is possible that SPRY proteins integrate both of these signals.

\section{Declaration of interest}

The authors declare that there is no conflict of interest that could be perceived as prejudicing the impartiality of this work.

\section{Funding}

This work is solely funded by A-Star (Singapore's Agency for Science Technology and Research) an agency of the Singapore Government. This work was supported by the Institute of Molecular and Cell Biology (Singapore) and A-Star (Singapore's Agency for Science Technology and Research). 


\section{Acknowledgements}

The authors thank the Agency of Science and Technology (A $\star$ STAR) of Singapore for financial support and Daniel Yim for generating the Sprouty proteins sequence alignment data.

\section{References}

Araki T, Nawa H \& Neel BG 2003 Tyrosyl phosphorylation of Shp2 is required for normal ERK activation in response to some, but not all, growth factors. Journal of Biological Chemistry 278 41677-41684.

Aranda S, Alvarez M, Turro S, Laguna A \& de la Luna S 2008 Sprouty2mediated inhibition of fibroblast growth factor signaling is modulated by the protein kinase DYRK1A. Molecular and Cellular Biology 28 5899-5911.

Bjorge JD, Jakymiw A \& Fujita DJ 2000 Selected glimpses into the activation and function of Src kinase. Oncogene 19 5620-5635.

Brown MD \& Sacks DB 2009 Protein scaffolds in MAP kinase signaling. Cellular Signalling 21 462-469.

Bundschu K, Walter U \& Schuh K 2007 Getting a first clue about SPRED functions. BioEssays 29 897-907.

Cabrita MA \& Christofori G 2008 Sprouty proteins; masterminds of receptor tyrosine kinase signaling. Angiogenesis 11 53-62.

Cabrita MA, Jaggi F, Widjaja SP \& Christofori G 2006 A functional interaction between Sprouty proteins and Caveolin-1. Journal of Biological Chemistry 281 29201-29212.

Casci T, Vinos J \& Freeman M 1999 Sprouty, an intracellular inhibitor of Ras signaling. Cell 96 655-665.

Chan G, Kalaitzidis D \& Neel BG 2008 The tyrosine phosphatase Shp2 (PTPN11) in cancer. Cancer Metastasis Reviews 27 179-192.

Chandramouli S, Yu CY, Yusoff P, Lao DH, Leong HF, Mizuno K \& Guy GR 2008 Tesk1 interacts with Spry2 to abrogate its inhibition of ERK phosphorylation downstream of receptor tyrosine kinase signaling. Journal of Biological Chemistry 283 1679-1691.

Chow SY, Yu CY \& Guy GR 2009 Sprouty2 interacts with protein kinase C $\delta$ and disrupts phosphorylation of protein kinase D1. Journal of Biological Chemistry 284 19623-19636.

DaSilva J, Xu L, Kim HJ, Miller WT \& Bar-Sagi D 2006 Regulation of Sprouty stability by Mnk-1 dependent phosphorylation. Molecular and Cellular Biology 26 1898-1907.

DeMille MM, Kimmel BE \& Rubin GM 1996 A Drosophila gene regulated by rough and glass shows similarity to ena and VASP. Gene 183 103-108.

Dhillon AS, Hagan S, Rath O \& Kolch W 2007 MAP kinase signaling pathways in cancer. Oncogene 26 3279-3290.

Fiete DJ, Beranek MC \& Baeziger JU 1998 A cysteine-rich domain of the mannose receptor mediates GalNac-4-SO4 binding. PNAS 95 2089-2093.

Fong CW, Leong HF, Wong ES, Lim J, Yusoff P \& Guy GR 2003 Tyrosine phosphorylation of Sprouty2 enhances its interaction with $\mathrm{c}-\mathrm{Cbl}$ and is crucial for its function. Journal of Biological Chemistry 278 33456-33464.

Guy GR, Wong ES, Yusoff P, Chandramouli S, Lo TL, Lim J \& Fong CW 2003 Sprouty; how does the branch manager work? Journal of Cell Science 116 3061-3068.

Hacohen N, Kramer S, Sutherland D, Hironi Y \& Krasnow MA 1998 Sprouty encodes a novel antagonist that patterns apical branching of the Drosophila airways. Cell 92 253-263.

Hadari YR, Kouhara H, Lax I \& Schlessinger J 1998 Binding of Shp2 tyrosine phosphatasae to FRS2 is essential for fibroblast growth factor-induced PC12 differentiation. Molecular and Cellular Biology 18 3966-3973.

Haglund K, Schmidt MH, Wong ES, Guy GR \& Dikic I 2005 Sprouty2 acts on $\mathrm{Cbl} / \mathrm{CIN} 85$ interface to inhibit epidermal growth factor receptor downregulation. EMBO Reports 6 635-641.

Hall AB, Jura N, DaSilva J, Jang YJ, Gong D \& Bar-Sagi D 2003 hSpry2 is targeted to the ubiquitin-dependent proteasome pathway by c-Cbl. Current Biology 13 308-314.
Hanafusa H, Torii S, Yasunaga T, Matsumoto K \& Nishida E 2004 Shp2, and $\mathrm{SH} 2$-containing protein-tyrosine phosphatase, positively regulates receptor tyrosine signaling by dephosphorylating and inactivating the inhibitor Sprouty. Journal of Biological Chemistry 279 22992-22995.

$\mathrm{Hu}$ J \& Hubbard SR 2005 Structural characterization of a novel Cbl phosphotyrosine recognition motif in the APS family of adaptor proteins. Journal of Biological Chemistry 280 18943-18949.

Hu J, Reyes-Cruz G, Goldsmith PK \& Spiegel AM 2001 The Venus's-flytrap and cysteine-rich domains of the human $\mathrm{Ca}^{2+}$ receptor are not linked by disulfide binds. Journal of Biological Chemistry 276 6901-6904.

Impagnatiello MA, Weitzer S, Gannon G, Compagni G, Cotton M \& Christofori G 2001 Mammalian sprouty-1 and -2 are membrane-anchored phosphoprotein inhibitors of growth factor signaling in endothelial cells. Journal of Cell Biology 152 1087-1098.

Jarvis LA, Toering SJ, Simon MA, Krasnow MA \& Smith-Bolton RK 2006 Sprouty proteins are in vivo targets of Corkscrew/SHP-2 tyrosine phosphatases. Development 133 1133-1142.

Kato R, Nonami A, Taketomi T, Wakioka T, Kuroiwa A, Matsuda Y \& Yoshimura A 2003 Molecular cloning of mammalian Spred-3 which suppresses tyrosine kinase-mediated Erk activation. Biochemical and Biophysical Research Communications 302 767-772.

Kim HJ \& Bar-Sagi D 2004 Modulation of signaling by Sprouty; a developing story. Nature Reviews. Molecular Cell Biology 6 441-450.

Kim HJ, Taylor LJ \& Bar-Sagi D 2007 Spatial regulation of EGFR signaling by Sprouty2. Current Biology 17 377-385.

King JA, Straffon AFL, D’Abaco GM, Poon CLC, Smith CM, Buchert M, Corcoran NM, Hall NE, Callus BA, Sarcevic B et al. 2005 Distinct requirements for the Sprouty domain for functional activity of Spred proteins. Biochemical Journal 388 445-454.

King JA, Corcoran NM, D'Abaco GM, Straffon AF, Smith CT, Poon CLC, Buchert M, Stacey I, Hall NE, Lock P et al. 2006 Eve-3: a liver enriched suppressor of Ras/MAPK signaling. Journal of Hepatology 44 758-767.

Lao DH, Chandramouli S, Yusoff P, Saw TY, Tai LP, Yu CY, Leong HF \& Guy GR 2006 A Src homology 3-binding sequence on the C terminus of Sprouty2 is necessary for inhibition of the Ras/ERK pathway downstream of fibroblast growth factor receptor stimulation. Journal of Biological Chemistry 281 29993-30000.

Lao DH, Yusoff P, Chandramouli S, Philp RJ, Fong CW, Jackson RA, Saw TY, Yu CY \& Guy GR 2007 Direct binding of PP2A to Sprouty2 and phosphorylation changes are a prerequisite for ERK inhibition downstream of fibroblast growth factor receptor stimulation. Journal of Biological Chemistry 282 9117-9126.

Leeksma OC, Van Achterberg TA, Tsumara Y, Toshima J, Eldering E, Kroes WG, Mellink C, Spaargaren M, Mizuno K, Pannekok H et al. 2002 Human sprouty 4 a new ras antagonist on $5 \mathrm{q} 31$, interacts with the dual specificity kinase TESK1. European Journal of Biochemistry 269 2546-2556.

Li X, Wheldon L \& Heath J 2003 Sprouty: a controversial role in receptor tyrosine kinase signaling pathways. Biochemical Society Transactions $\mathbf{6}$ 1445-1446.

Li X, Brunton VG, Burgar HR, Wheldon LM \& Heath JK 2004 FRS2dependent $\mathrm{Src}$ activation is required for fibroblast growth factor receptor-induced phosphorylation of Sprouty and Suppression of ERK activity. Journal of Cell Science 117 6007-6017.

Lim J, Wong ES, Ong SH, Yusoff P, Low BC \& Guy GR 2000 Sprouty proteins are targeted to membrane ruffles upon growth factor receptor tyrosine kinase activation. Identification of a novel translocation domain. Journal of Biological Chemistry 275 32837-32845.

Lim J, Yusoff P, Wong ES, Chandramouli S, Lao DH, Fong CW \& Guy GR 2002 The cysteine-rich sprouty translocation domain targets mitogen-activated protein kinase inhibitory proteins to phosphatidyl 4,5,-bisphosphate in plasma membranes. Molecular and Cellular Biology 22 7953-7966.

Lupher ML, Songyang Z, Shoelson SE, Cantley LC \& Band H 1997 The Cbl phosphotyrosine-binding domain slects a D(N/D)XpY motif and binds to the Tyr 292 negative regulatory phosphorylation site of Zap-70. Journal of Biological Chemistry 272 33140-33144. 
Mason JM, Morrison DJ, Bassit B, Dimri M, Band H, Licht JD \& Gross I 2004 Tyrosine phosphorylation of Sprouty proteins regulates their ability to inhibit growth factor signaling: a dual feedback loop. Molecular and Cellular Biology 15 2176-2188.

Mason JM, Morrison DJ, Basson MA \& Licht JD 2006 Sprouty proteins; multifaceted negative-feedback regulators of receptor tyrosine kinase signaling. Trends in Cell Biology 16 45-54.

de Maximy AA, Nakatake Y, Moncada S, Itoh N, Thiery JP \& Bellusci S 1999 Cloning and expression patterns of a mouse homologue of Drosophila sprouty in the mouse embryo. Mechanisms of Development 81 75-88.

Meng W, Sawasdikosol S, Burakoff SJ \& Eck MJ 1999 Structure of the aminoterminal domain of $\mathrm{Cbl}$ complexed to its binding site on ZAP-70 kinase. Nature 398 84-90.

Minowada G, Jarvis LA, Chi CL, Neubuser A, Sun X, Hacohen N, Krasnow MA \& Martin GR 1999 Vertebrate sprouty genes are induced by FGF signalling and can cause chondroplaysia when overexpressed. Development $1264465-4475$.

Mott HR, Carpenter JW, Zhong S, Ghosh S, Bell RM \& Campbell SL 1996 The solution structure of the Raf-1 cysteine-rich domain: a novel Ras and phospholipid binding site. PNAS 93 8312-8317.

Murphy LO \& Blenis J 2006 MAPK signal specificity: the right place at the right time. Trends in Biochemical Sciences 31 268-275.

Nadeau RJ, Toher JL, Yang X, Kovalenko D \& Friesel R 2006 Regulation of Sprouty2 stability by mammalian Seven-in Absentia Homolog 2. Journal of Cellular Biochemistry 100 151-160.

Ng C, Jackson RA, Buschdorf JP, Sun Q, Guy GR \& Sivaraman J 2008 Structural basis for a novel intrapeptidyl H-bond and reverse binding of a c-Cbl-TKB domain substrates. EMBO Journal 27 804-816.

Nonami A, Taketomi T, Kimura A, Saeki K, Takaki H, Sanada T, Taniguchi K, Harada M \& Yoshimura A 2005 The Sprouty-related protein, Spred-1, localizes in a lipid raft/caveola and inhibits ERK activation in collaboration with caveolin-1. Genes to Cells 10 887-895.

Ojalo JRM, Pikkarainen T, Tuuttila A, Sandalova T \& Tryggvason K 2007 Crystal structure of the cysteine-rich domain of scavenger receptor MARCO reveals the presence of a basic and an acidic cluster that both contribute to ligand recognition. Journal of Biological Chemistry $\mathbf{2 8 2}$ 16654-16666.

Okada T, Hu C-D, Jin T-G, Kariya K-I, Yamawaki-Kataoka Y \& Kataoka T 1999 The strength of interaction at the Raf cysteine-rich domain is a critical determinant of response of Raf to Ras family small GTPases. Molecular and Cellular Biology 19 6057-6064.

Ozaki K, Miyazaki S, Tanimura S \& Kohno M 2005 Efficient suppression of FGF-2 induced ERK activation by the cooperative interaction among mammalian Sprouty isoforms. Journal of Cell Science 118 5861-5871.

Pearson G, Robinson F, Gibson TB, Xu BE, Karandikar M, Berman K \& Cobb M 2001 Mitogen-activated protein (MAP) kinase pathways: regulation and physiological functions. Endocrinology Reviews 22 153-183.

Peschard P, Fournier TM, Lamorte L, Naujokas MA, Band H, Langdon WY \& Park M 2001 Mutation of the c-Cbl TKB domain binding site on the Met receptor tyrosine kinase converts it into a transforming protein. Molecular Cell 8 995-1004.

Roux PP \& Blenis J 2004 ERK and p38 MAPK-activated protein kinases: a family of protein kinases with diverse biological functions. Microbiology and Molecular Biology Reviews 68 320-344.

Rubin C, Litvak V, Medvedovsky H, Zwang Y, Lev S \& Yarden Y 2003 Sprouty fine-tunes EGF signaling through interlinked positive and negative feedback loops. Current Biology 13 297-307.

Sasaki A, Taketomi T, Wakioka T, Kato R \& Yoshimura A 2001 Identification of a dominant negative mutant of Sprouty that potentiates fibroblast growth factor- but not epidermal growth factor-induced ERK activation. Journal of Biological Chemistry 276 36804-36808.

Sasaki A, Taketomi T, Kato R, Saeki K, Nonami A, Sasaki M, Kuriyama M, Saito N, Shibuya M \& Yoshimura A 2003 Mammalian Sprouty4 suppresses Ras-independent ERK activation by binding to Raf1. Nature Cell Biology $\mathbf{5}$ 427-432.
Schmidt MH \& Dikic I 2005 The $\mathrm{Cbl}$ interactome and its functions. Nature Reviews. Molecular Cell Biology 6 907-919.

Sweet SM, Mardakheh FK, Ryan KJP, Langton AJ, Heath JK \& Cooper HJ 2008 Targeted online liquid chromatography electron capture dissociation mass spectrometry for the localization of sites of in vivo phosphorylation in human Sprouty2. Analytical Chemistry 80 6650-6657.

Tan YM, Cardinal J, Franks AH, Mun H-C, Lewis N, Harris LB, Prins JB \& Connigrave AD 2003 Autosomal dominant hypocalcemia: a novel activating mutation (E604K) in the cysteine-rich domain of the calcium-sensing receptor. Journal of Clinical Endocrinology and Metabolism 88 605-610.

Tanaka Y, Tanaka N, Saeki Y, Tanaka K, Murakami M, Hirano T, Ishii N \& Sugamura K 2008 c-Cbl-dependent monoubiquitination and lysosomal degradation of gp130. Molecular and Cellular Biology 28 4805-4818.

Tartaglia M, Mehler EL, Goldberg R, Zampino G, Brunner HG, Kremer H, van der Burgt I, Crosby AH, Ion A, Jeffrey S et al. 2001 Mutations in PTPN11, encoding the protein tyrosine phosphatase SHP-2, cause Noonan syndrome. Nature Genetics 29 465-468.

Tefft JD, Lee M, Smith S, Leinwand M, Zhao J, Bringas P Jr, Crowe DL \& Warburton D 1999 Conserved function of mSpry-2, a murine homolog of Drosophila sprouty, which negatively modulates respiratory organogenesis. Current Biology 9 219-222.

Thien CB \& Langdon WY 2005 c-Cbl and Cbl-b ubiquitin ligase: substrate diversity and the negative regulation of signaling responses. Biochemical Journal 391 153-166.

Thum T, Gross C, Fiedler J, Fischer T, Kissler S, Bussen M, Galuppo P, Just S, Rottbauer W, Frantz S et al. 2008 MicroRNA-21 contributes to myocardial disease by stimulating MAP kinase signalling in fibroblasts. Nature 456 980-986.

Torii S, Kusakabe M, Yamamoto T, Maekawa M \& Nishida E 2004 Sef is a spatial regulator for Ras/Map kinase signaling. Developmental Cell 7 33-44.

Tsumura Y, Toshima J, Leeksma OC, Ohashi K \& Mizuno K 2005 Sprouty-4 negatively regulates cell spreading by inhibiting the kinase activity of testicular protein kinase. Biochemical Journal 387 627-637.

Ubersax JA \& Ferrell JE 2007 Mechanisms of speficity in protein phosphorylation. Nature Reviews. Molecular Cell Biology 8 530-541.

Wakioka T, Sasaki A, Kato R, Shouda T, Matsumoto A, Miyoshi K, Tsuneoka M, Komiya S, Baron R \& Yoshimura A 2001 Spred is a Sprouty-related suppressor of Ras signalling. Nature 412 647-651.

Wong ES, Lim J, Low BC, Chen Q \& Guy GR 2001 Evidence for direct interaction between Sprouty and Cbl. Journal of Biological Chemistry 2756 4128-4133.

Wong A, Lamothe B, Lee A, Schlessinger J \& Lax I 2002a FRS2 alpha attenuates FGF receptor signaling by Grb2-mediated recruitment of the ubiquitin ligase Cbl. PNAS 99 6684-6689.

Wong ES, Fong CW, Lim J, Yusoff P, Low BC, Langdon WY \& Guy GR $2002 b$ Sprouty 2 attenuates epidermal growth factor receptor ubiquitylation and endocytosis, and consequently enhances Ras/ERK signaling. EMBO Journal 21 4796-4808.

Zhao Z, Gruszczynska-Biegala J, Cheuvront T, Yi H, von der Mark H, von der Mark K, Kaufman SJ \& Zolkiewska A 2004 Interaction of the disintegrin and cysteine-rich domains of ADAM12 with integrin alpha7beta1. Experimental Cell Research 298 28-37.

Zhou Y, Tzeng W-P, Ye Y, Huang Y, Li S, Chen Y, Frey TK \& Yang JJ 2009 A cysteine-rich metal-binding domain from rubella virus non-structural protein is essential for viral protease activity and virus replication. Biochemical Journal 417 477-483

Received in final form 27 April 2009

Accepted 7 May 2009

Made available online as an Accepted Preprint

7 May 2009 